\title{
Local versus global minima in visual pattern completion
}

\author{
FRANS BOSELIE \\ University of Nijmegen, Nijmegen, The Netherlands
}

\begin{abstract}
The phenomenon of visual completion poses two questions: (1) When will a figure be seen as being in front of another, as opposed to being one of several nonoverlapping figures in the same plane? And (2) exactly what will be seen behind the occluding figure? Buffart, Leeuwenberg, and Restle $(1981,1983)$ recently argued that both questions could be answered by coding theory. Coding theory assumes that a global minimum principle is operative in perception, and that perception will result in an interpretation that can be represented by a code with a minimal information load. Contrary to this view, it is claimed here that only those completion and mosaic interpretations that exploit the local advantage of continuity of sides at points of intersection or at the common contour will be perceptually salient. When both a locally simple completion interpretation and a locally simple mosaic interpretation can be made, preference will be stronger for the type of interpretation that is globally more simple. To refute Buffart et al.'s claim and to test the hypotheses formulated above, an experiment was performed in which patterns similar to those used by Buffart et al. were presented to subjects, who traced the contours of the figures they saw. Only a very small proportion of the responses given by the subjects were as predicted by coding theory. The data, on the other hand, supported the idea that a global minimum principle operates within the constraints of locally minimal descriptions.
\end{abstract}

When looking around, we see all kinds of things that are partly occluded by other objects in front of them. Very often we have a definite impression of what exactly is hidden behind the objects seen in front. This phenomenon has been given several names: interposition (Chapanis \& McCleary, 1953), phenomenal overlapping (Dinnerstein \& Wertheimer, 1957), amodal completion (Kanizsa, 1975), occlusion (Gibson, 1979), and figural completion (Buffart, Leeuwenberg, \& Restle, 1981, 1983). Two questions raised by this phenomenon are: (1) When will one figure in front of another be seen, as opposed to two nonoverlapping figures in the same plane? (2) What will be seen behind the occluding object? Buffart et al. (1981) recently proposed a solution, arguing that both questions could be answered by Leeuwenberg's coding theory (Leeuwenberg, 1969, 1971; Restle, 1982).

\section{Coding Theory of Visual Completion}

Coding theory assumes that a global minimum principle - that is, a minimum principle that applies to a pattern as a whole-is operative in perception, and that perception of a pattern will result in an interpretation that can be represented by a code with minimal information load. Leeuwenberg's coding system $(1969,1971)$ provides a method of describing interpretations of visual patterns in such a way that they can be ordered in terms of infor-

I am indebted to Emanuel Leeuwenberg for his valuable comments on an earlier draft of this paper, and to Hans Buffart, who helped me in coding many of the patterns presented here. Address correspondence to Frans Boselie, Department of Experimental Psychology, University of Nijmegen, P. O. Box $9104,6500 \mathrm{H}$. E. Nijmegen, The Netherlands.
Table 1 Examples of Coding Rules

\begin{tabular}{llll}
\hline Coding Rule & Primitive Code & Reduced Code & $I$ \\
\hline Iteration & a a & $2 *(a)$ & 2 \\
Symmetry & a b b a & SYM[a b] & 3 \\
Symmetry & a b c b a & SYMM[a b. (c)] & 4 \\
Distribution & a b a c & $<$ a $><$ b c $\rangle$ & 3 \\
Continuation & a a $\cdots \infty$ & @*(a) & 1 \\
\hline
\end{tabular}

Note $-I=$ information load.

mation load. Because his coding system has been presented in detail in several recent publications (Boselie \& Leeuwenberg, 1985; Buffart et al., 1981; Leeuwenberg \& Buffart, 1984), it will be outlined here only briefly. In Leeuwenberg's system, an interpretation of a visual pattern is represented by a series of symbols, or a primitive code. In the case of a two-dimensional line pattern, a primitive code is made by tracing the contours of the pattern and successively noting the lengths of line elements $(k, 1, m, \ldots)$ and the angles between them $(a, b, c, \ldots)$. If a primitive code shows redundancy, it can be rewritten in a simpler form by means of the syntactic coding rules of the system. Table 1 presents examples of five coding rules.

These coding rules correspond to regularities such as repetition, mirror symmetry, rotation symmetry, and alternation. If possible, the simplified code can be reduced further in this way. When the code cannot be further reduced, the result is called a final code. The number of rules employed, together with the remaining irreducible elements in a code, is conceived of as a measure of the information load (I), or complexity, of a code. Final codes 


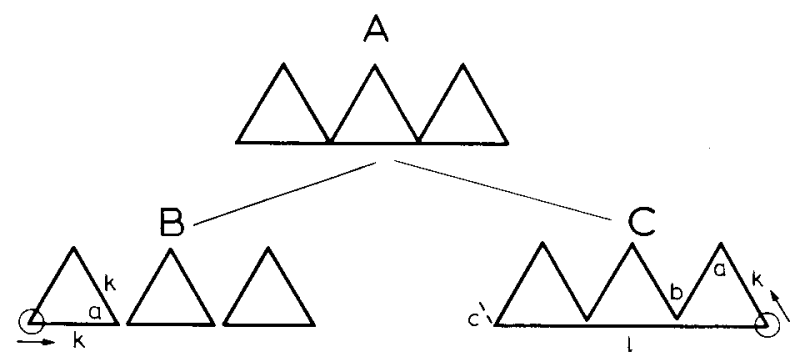

Figure 1. A pattern (A) with two possible interpretations (B and C). Coding starts at the small circle and proceeds in the direction indicated by the arrow. Coding theory predicts that interpretation $B$ will be the perceptually preferred one.

can be ordered according to $I$. A final code with the lowest structural information load is called a minimum code. The perception of a pattern will result in an interpretation that can be represented by a minimum code. Coding theory thus replaces the plethora of Gestalt principles with a single assumption: that the information load of what is actually perceived is lower than the information load of what could have been perceived. This is the formulation of the old law of Prägnanz (Koffka, 1935), also known as the minimum principle (Hochberg \& McAlister, 1953) within coding theory.

As a case in point, Figure 1 illustrates the coding of two interpretations of a simple pattern. The codes are simplified. The code elements that contribute to $I$ are indicated by dots.

A

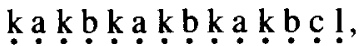

$(I=14)$

which, by iteration, equals

$$
3^{*}(\mathrm{k} \text { a } \mathrm{k} \text { b }) \text { c } 1 \text {, }
$$$$
(I=7)
$$

which, by distribution, equals

$$
3^{*}(<\underline{\mathrm{k}}><\underline{\mathrm{a}} \mathbf{\mathrm { b }}>\mathbf{\mathrm { c }} \mathbf{\mathrm { l }},
$$

which is a final code.

On the basis of the $I$ values of these final codes, coding theory predicts that Figure 1A will be preferentially interpreted as three triangles.

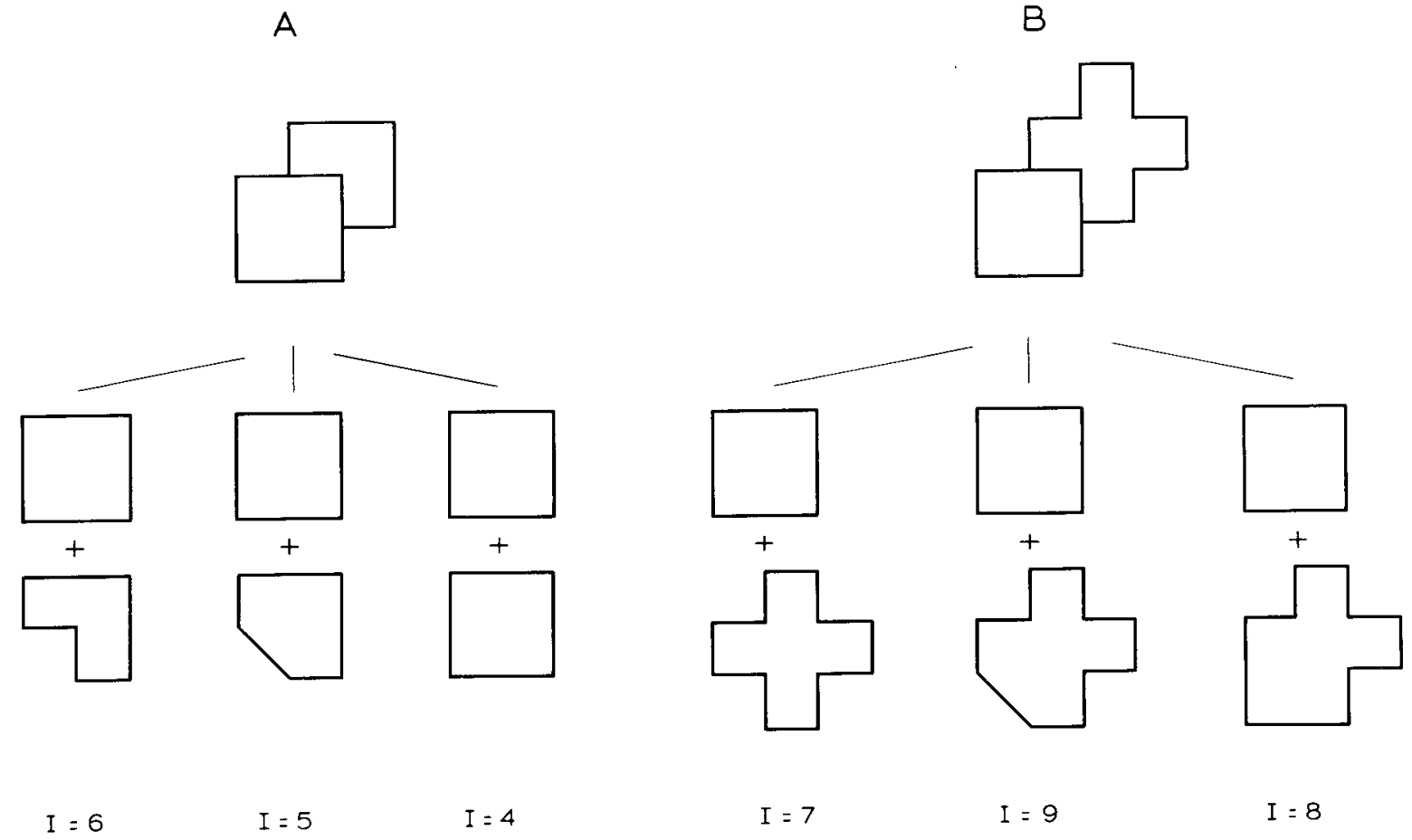

Figure 2. Two patterns (A and B), each with three possible interpretations: a mosaic interpretation and two different completion interpretations. The information load, $I$, of the minimum code, by which the interpretation can be represented, is given for each interpretation. Coding theory predicts that the interpretation with the lowest $I$ value will be perceptually preferred. 
During the past 10 years, Leeuwenberg and his coworkers have reported on a number of experiments that tested predictions based on the global minimum principle. The experiments related to the judged complexity of patterns (Leeuwenberg, 1969, 1971), subjective contours (Van Tuijl \& Leeuwenberg, 1979, 1982), the perception of motion (Restle, 1979), embeddedness (Van Tuijl, 1980), the effect of context on the perception of form (Collard \& Leeuwenberg, 1981), assimilation and contrast (Leeuwenberg, 1982), the perception of foreground and background (Boselie \& Leeuwenberg, 1986; Leeuwenberg \& Buffart, 1984), and the perceptual attractivity of patterns (Boselie \& Leeuwenberg, 1985). On the whole, the results of these experiments fit in with the ordering of interpretations according to perceptual simplicity, as inferred from the system of coding rules. They also lend support to the view that the perceptual preference for certain interpretations is based on a global minimum principle.

Returning to the two questions raised by the phenomenon of perceptual completion, the answers provided by coding theory are clear-cut. First, if a subject is shown a pattern that can be interpreted either as a mosaic or as one figure occluding another one, he or she will see the interpretation that, when coded, yields the lowest information load. Second, if a figure is perceptually completed behind an occluding one, the subject will see the completion that yields the lowest information load for the pattern as a whole (see Figure 2 for an illustration). When two different interpretations of a pattern, as represented by final codes, have the same minimal information load, coding theory predicts that the pattern will be perceptually ambiguous and that responses will be distributed over the equivalent interpretations. According to Buffart et al. (1981), such a prediction of ambiguity provides a strong test of coding theory.

To test their predictions, Buffart et al. (1981) designed 25 patterns (see Figure 3), each of which consisted of a square and one or more additional two-dimensional figures. In two experiments, the subjects $(N=30$ and $N=74$, respectively) were asked to look at each pattern carefully and to trace the contour of the figure or figures that, in their perception of the pattern, accompanied the square. Each of the 25 patterns had two or more possible interpretations: the mosaic interpretation and one or more examples of completion of a figure behind an occluding one. Buffart et al. prepared minimum codes for the mosiac interpretations and for the different completion interpretations. Of the 25 patterns, 14 had completions with a lower information load than the mosaic interpretation, 9 had equal information loads (the authors erroneously give the numbers 16 and 7 , respectively), and the other 2 had mosaic interpretations with minimal information loads. The results of the experiments were as follows. When completions had lower information loads than mosaics, $96 \%$ of the subjects produced completions; when the information loads were equal, $45 \%$ of the subjects produced completions; and when mosaics had lower information
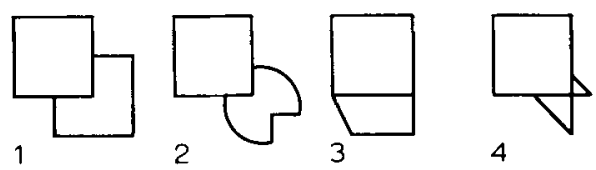

4
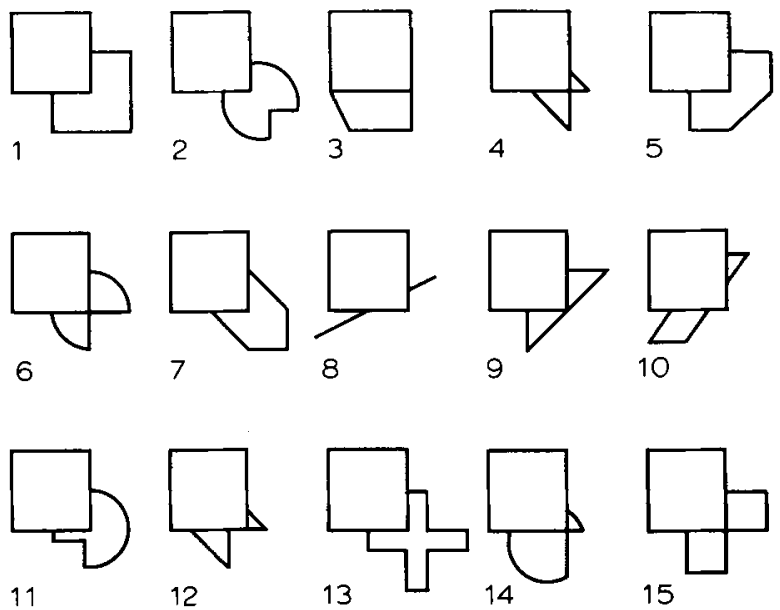

11

12

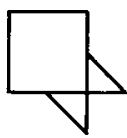

16

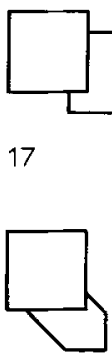

22
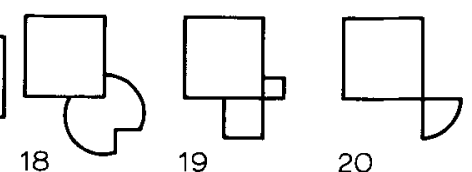

20

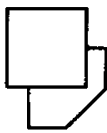

21

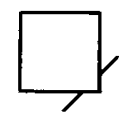

23
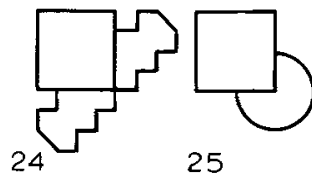

25

Figure 3. Patterns used by Buffart et al. (1981).

loads than completions, only $10 \%$ of the subjects produced completions. Buffart et al. claimed complete success for their combination of a coding system with the global minimum principle in predicting perceptual pattern completion, a task at which other theories had failed.

\section{Criticisms of a Global Minimum Principle}

Hatfield and Epstein (1985) drew attention to an important aspect of all investigations in which a minimum principle is put to an empirical test. They rightly observed that a straightforward test of a minimum principle is not possible because, in order to test the minimum principle, one first of all has to know exactly what resources the perceptual system can bring to bear to represent the structure of a pattern. Only then would one be in a position to test whether the system actually did prefer the simplest possible interpretation of a pattern.

Practically, the situation is as follows. Coding theorists make a great number of assumptions as to the competence of the perceptual system to represent the structure of patterns. They also assume that the perceptual process will always result in the simplest possible interpretation for a pattern as a whole. This situation thus necessarily implies that investigators are actually testing the psychophysical adequacy of their own sets of coding rules and measures of simplicity. Hatfield and Epstein (1985) argued that this is not an uncommon situation in the logic 
of experimentation, which is by no means fatal, provided that the postulated set of coding rules displays long-run empirical fruitfulness. As the published evidence bears out, many phenomena of perceptual organization can indeed be described by Leeuwenberg's $(1969,1971)$ coding sytsem, and the system will be improved and augmented, if necessary, with each increment of experimental knowledge.

Apart from the fact that a straightforward test of a global minimum principle is not possible, some students of perception do not believe that a global minimum principle is operative in perception at all. Rock (1983, chap. 6) explored the basis of a preference for one interpretation over another. His answer was that the perceptual system sought interpretations that explained seeming coincidences and regularities that were otherwise implicit in the nonpreferred interpretations. He gave a great number of examples in support of this coincidence principle, and then concluded: "As to the simplicity doctrine, I am inclined to rule it out on the basis of the evidence considered in this chapter"' (p. 164). However, Boselie and Leeuwenberg (1986) showed that not even one of Rock's examples refutes the global minimum principle if Leeuwenberg's coding system is used to quantify the complexity of interpretations. I will give one example.
There is a clear preference to perceive Figure $4 \mathrm{~A}$ as one figure in front of and partially occluding another figure. When the completion effect occurs, the percept is simpler than when the figure is seen as a mosaic of two figures in one plane. So the preference can be explained by the operation of a minimum principle. But Rock (1983) argued that Figure 4A was a special case. He presented Figure $4 \mathrm{~B}$ as an example of the more general case, in which the two regions are irregular shapes. When the completion effect occurred in this type of pattern, the resulting percept was, in his opinion, not simpler than when it was seen as coplanar. So a minimum principle could not explain the preferred perceptual outcome. But the completion interpretation did account for the coincidental feature of the pattern - that is, the meshing of part of the boundaries of both regions along the common contour. Rock therefore concluded that Figure 4B supported the coincidence principle.

To refute Rock's (1983) claim, I determined the minimum codes to represent three interpretations of Figure $4 \mathrm{~B}$ : (1) the mosaic interpretation (region a next to region b; Figure 4C); (2) one interposition interpretation (region a in front of region b; Figure 4D); and (3) the other interposition interpretation (region $b$ in front of region $a$; Figure 4E). The contours of regions $a$ and $b$ can be
A

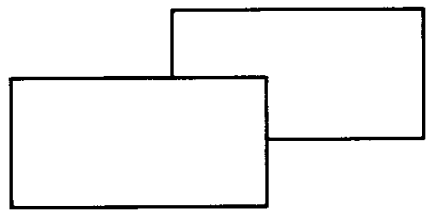

C

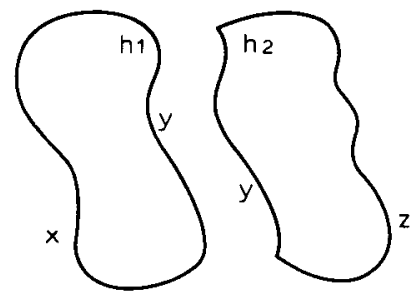

$I=(x+y+z)+(y+2)$
B

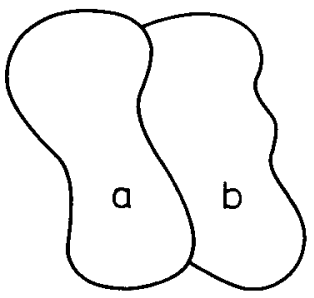

$\mathrm{D}$

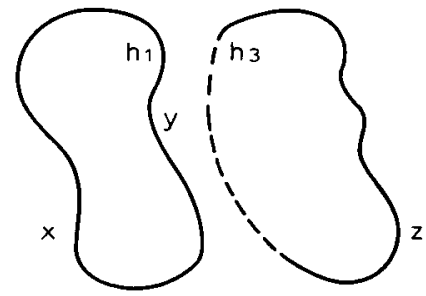

$I=(x+y+z)+2$
F

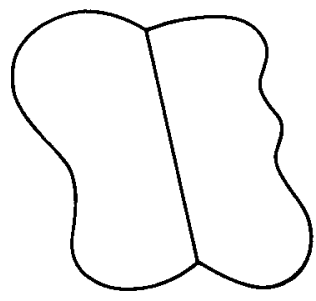

$E$

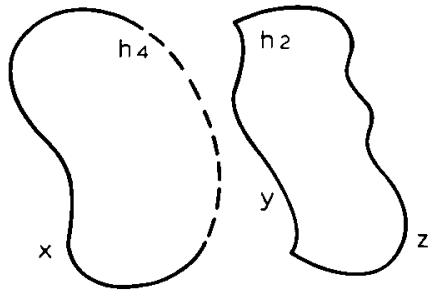

$I=(x+y+z)+2$

Figure 4. A: This pattern is preferentially perceived as two regular shapes. B: The more general case, in which the two regions are irregular (from Rock, 1983). C: The coding of the mosaic interpretation of B (region a next to region b). D: The coding of a completion interpretation of $B$ (region a in front of region b). E: The coding of the other completion interpretation of $B$ (region $b$ in front of region a). F: A pattern that results in a less robust completion effect. 
divided into three parts: the parts of the contours that regions $\mathrm{a}$ and $\mathrm{b}$ have in common (symbolized by $y$ ), the parts of the contours that belong exclusively to region a or b (symbolized by $x$ and $z$, respectively), and the angles by which these parts are connected ( $h 1$ through $h 4$ ). As shown in these figures, the information load of a completion interpretation of irregular patterns, such as that in Figure 4B, is smaller than the information load of the mosaic interpretation. This is because the contour of the occluded figure in a completion interpretation can be completed in a simple, regular way (see Boselie \& Leeuwenberg, 1986, for details). An exception occurs for those patterns for which $I y=0$. In those cases, in which the common contour is a simple straight line (see Figure 4F for an example), all interpretations carry the same amount of information. Coding theory therefore predicts that the completion effect in a pattern such as that shown in Figure $4 \mathrm{~F}$ will be weak. However, because the information load of the common contour is greater than zero, Figure 4B will preferentially be interpreted as being one figure in front of and partially occluding another (when region a is symmetrical and region $b$ is not, region a will be seen in front of region b). These predictions are in keeping with the phenomenal impression these patterns make. On the other hand, Figure 4F poses a problem for the coincidence principle because, in this pattern, too, contours of the regions coincide. In summary, I conclude that
Figure 4B, contrary to Rock's claim, does not give evidence against a global minimum principle.

Other patterns that provide phenomenological evidence against a global minimum principle have been presented by Kanizsa $(1975,1985,1986)$. Figure 5 gives an example. The central part of Figure 5A is easily seen as an irregular polygon that is partially covered by two squares, whereas, in principle, it can be interpreted as a regular octagon. The perceptual preference for an irregular polygon over a regular one seems to be at odds with a global minimum principle. However, when one makes minimal codes of these two interpretations, one finds that the codes have the same amount of structural information. Although it may seem surprising that the code representing the interpretation that contains the irregular polygon has the same information load as the code representing the regular octagon, it is a direct consequence of the global minimum principle as applied by Buffart et al. (1981). In their coding system, every length and angle of a pattern is described in the context of all other lengths and angles, irrespective of whether they form part of one contour or belong to the contours of different surfaces. The simplicity of the code that represents the octagon is based on the regularity of the octagon. The code that represents the irregular polygon is simple because the $90^{\circ}$ angles of the polygon are described as identical to the angles of the occluding squares, and because the length of the occluding

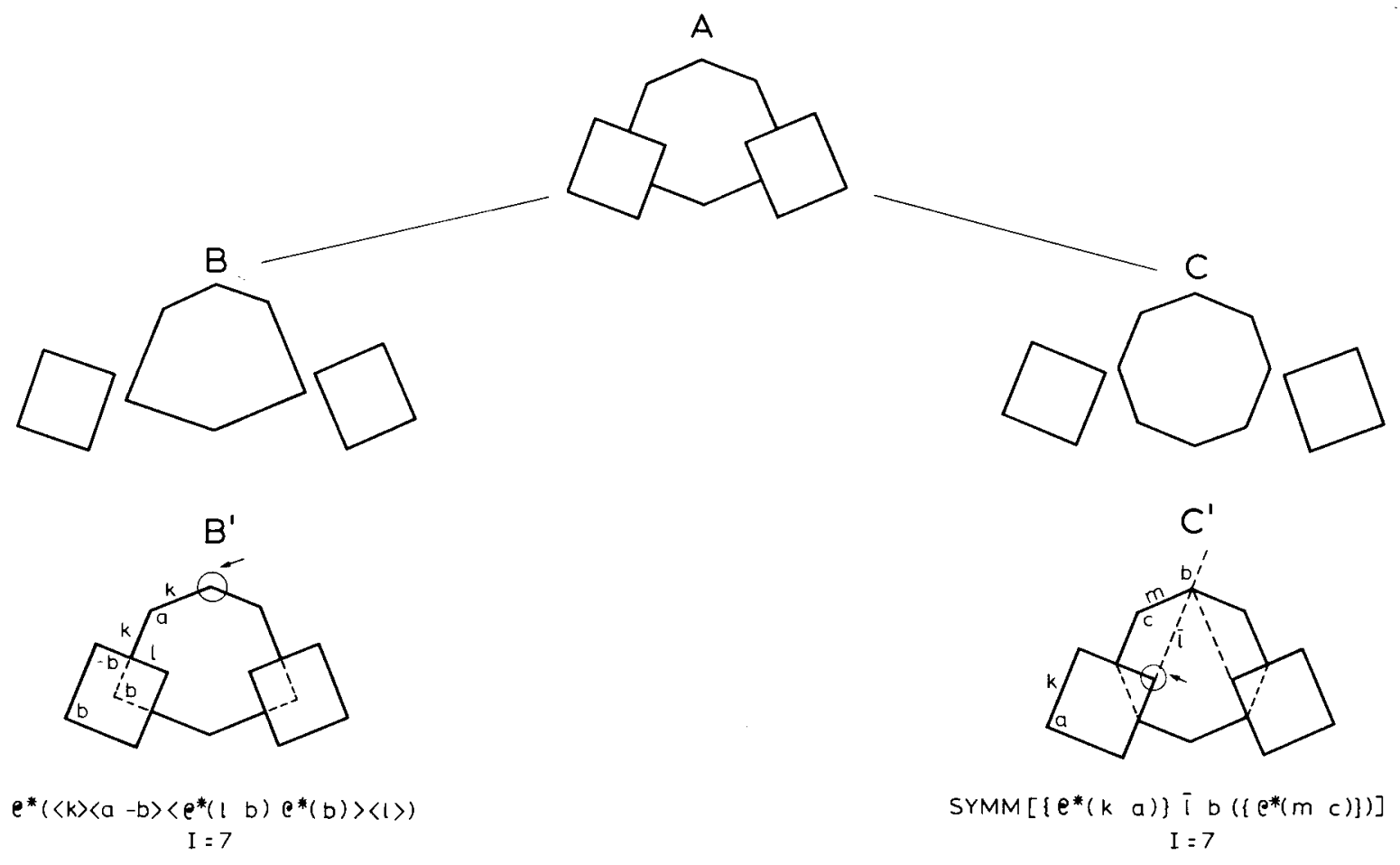

Figure 5. A: A line drawing of a pattern presented by Kanizsa (1985). B and C: Two interpretations of A. B' and C': The minimum codes, made according to Buffart et al.'s (1981) coding procedure, which represent interpretations B and C. Both interpretations can be represented by codes with the same amount of information $(I=7)$. The codes are written in the notational form used in the most recent publications by Leeuwenberg (Boselie \& Leeuwenberg, 1985, 1986; Leeuwenberg \& Buffart, 1984). 
A

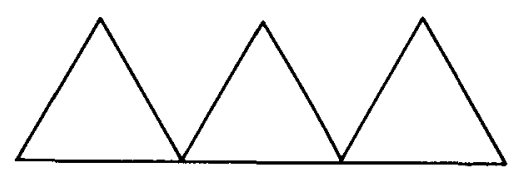

$\triangle \triangle \triangle$

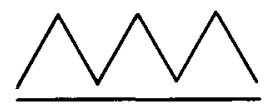

$\mathrm{I}=4$

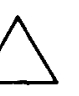

.

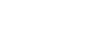

$$
I=6
$$

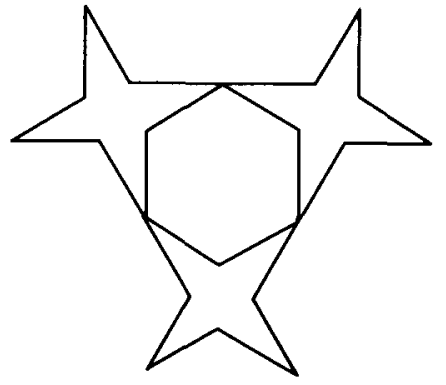

B

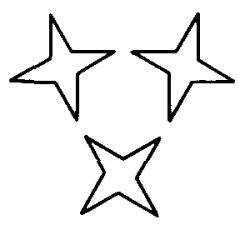

$I=6$

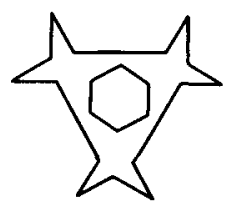

$\mathrm{I}=8$

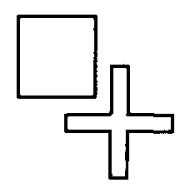

$I=7$
C

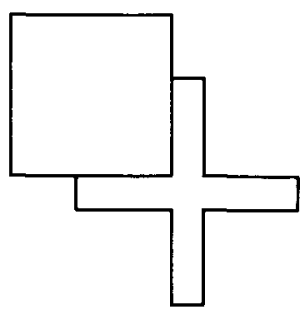

Figure 6. Three patterns, each with two possible interpretations. A looks like three triangles; B, like three stars; and C, like a cross next to a square. All these interpretations are incompatible with the rule of good continuation.

contours of the square are identical to the lengths of the occluded parts of the irregular polygon. Leeuwenberg's $(1969,1971)$ coding language exploits these regularities when making a minimal code of an interpretation in a clever way. In other words, according to coding theory, Figure $5 \mathrm{~A}$ is perceptually ambiguous, and the theory therefore does not prohibit one from seeing an irregular polygon in Figure 5A.

Like Rock (1983), Kanizsa (1985) stressed the role of good continuation in perceptual organization. He believed that a minimum principle applied only to local regions of a pattern, and that perceptual organization would not be influenced in a decisive way by the demands of regularity made by a pattern as a whole. However, good continuation as a general rule of perceptual organization is disproved by many examples, some of which are given in Figure 6. Figure 6A looks like three triangles and Figure 6B looks like three stars. Figure 6C does not contain a continuation behind the square, but is easily seen as a cross and a square. All these interpretations are incompatible with good continuation as a general principle of perceptual organization. They are, however, fully in keeping with a global minimum principle as specified by Leeuwenberg's $(1969,1971)$ coding theory, as demonstrated by the information loads of the corresponding codes (see Figure 6).

In summary, my conclusion is that the attacks on the global minimum principle have not been effective because they have not been based on a well-defined metric of simplicity of interpretations. A refutation of Buffart et al.'s (1981) claim that a global minimum principle is operative in perception can be based only on the application

of their own set of coding rules. The aim of the research reported here is to do just that. Numerous counterexamples to their coding theory will be provided. Most counterexamples will exploit the strong tendency to continue directions perceptually. However, I do not intend to present a definitive alternative theory in which "good continuation" is the core explanatory principle. My main aim is to undermine Buffart et al.'s claim that they solved the problems posed by the perceptual organization of visual overlap.

A
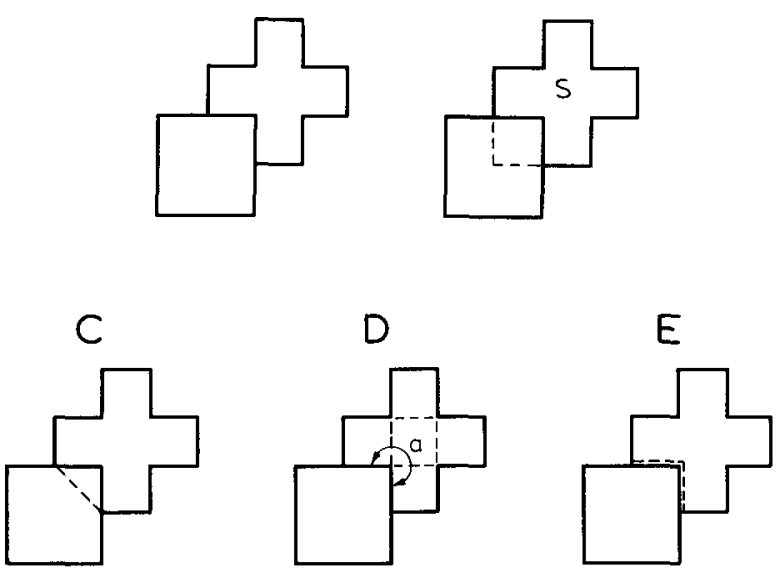

Figure 7. A pattern (A) with two completion interpretations (B and $C$ ) and two mosaic interpretations ( $D$ and $E$ ). Interpretations $B$ and $D$ both exploit the advantage of good continuation, whereas $C$ and $E$ do not. 


\section{Local Minima Constrain Global Ones}

The Gestalt principle of good continuation claims that contours are perceived as continuing smoothly along their original course. The principle, in fact, is the formulation of a locally operating minimum principle: A contour whose direction remains constant can be described more simply than one whose direction changes or one that terminates and is replaced by another contour. Note that the minimum principle is applied here strictly locally, with no regard for the consequences at more global levels. However, as already stated above, it has been repeatedly demonstrated that the principle of good continuation is not a general principle of perceptual organization: overall figural goodness is also an important factor in perceptual organization. Figure 6 gives some examples of forms in which good continuation on a local level is overridden by a more globally operating minimum principle.
Let us focus now on the role of good continuation in the perception of the kind of display studied by Buffart et al. (1981). Given such a pattern, two types of interpretations seem to be perceptually salient: a given display is seen either as a conglomerate of several surfaces in different planes, with some surfaces being partially occluded by other ones (an instance of completion), or as a conglomerate of several adjoining shapes in one plane (a mosaic). It seems to me that the main problem in the perception of this kind of display is the assigning of a satisfactory interpretation to the central region: the points of intersection and the common contour. My guess is that only those completion and mosaic interpretations that exploit the local advantage of continuity of sides in this central region of a pattern will be perceptually salient. When both a locally simple completion interpretation and a locally simple mosaic interpretation can be made, the prefer-

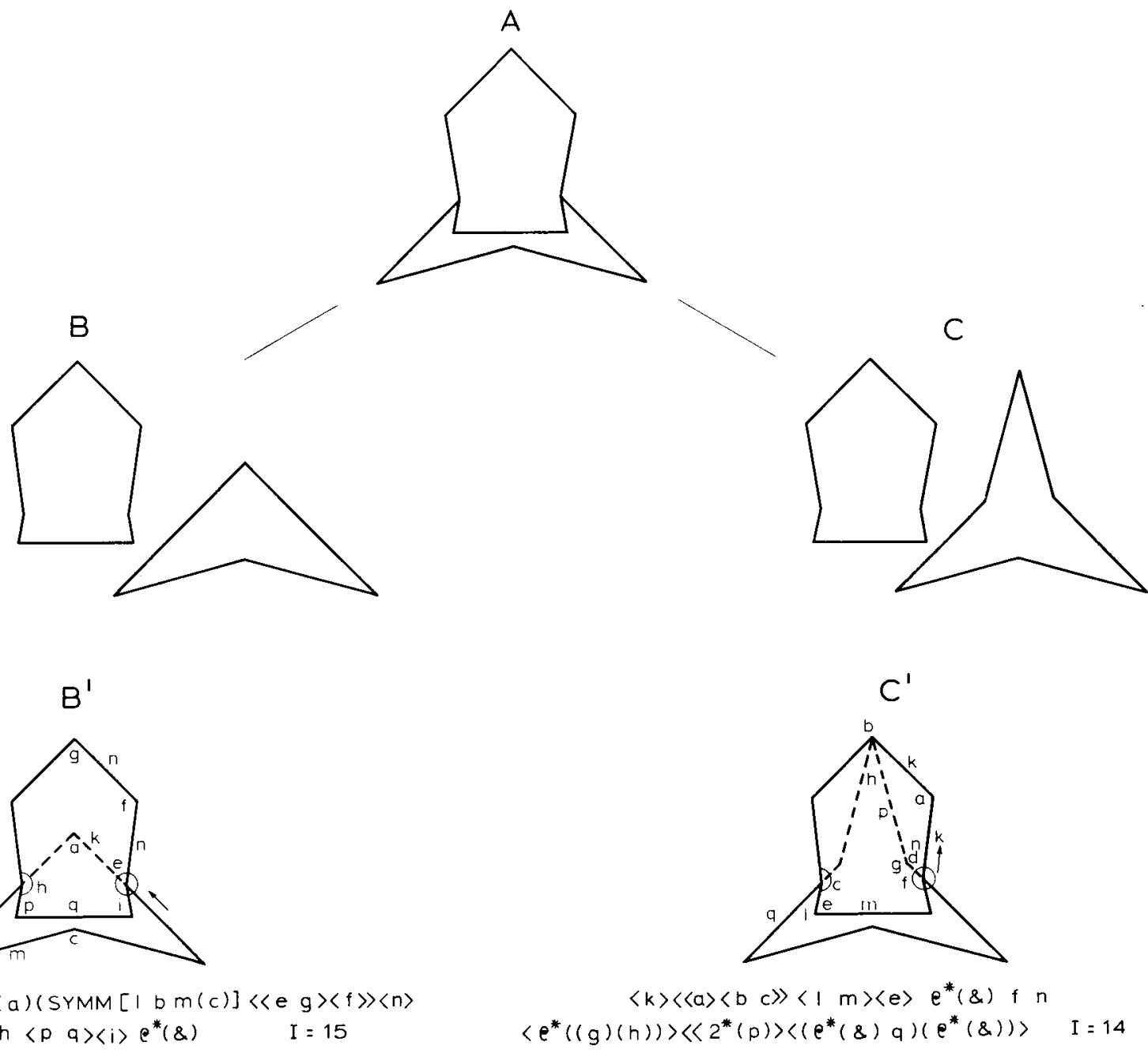

Figure 8. A pattern (A) with two completion interpretations (B and C). Both interpretations are much simpler than the mosaic interpretation. Interpretation B combines this high degree of global simplicity with good continuation, whereas interpretation $\mathbf{C}$ does not. $A$ will be preferentially interpreted as illustrated by $B$, and not as shown by $C$. $B^{\prime}$ and $C^{\prime}$ are the minimum codes, made according to Buffart et al.'s (1981) coding procedure, which represent interpretations $B$ and C. 
ence will be stronger for the interpretation that is globally more simple. In that way, both local and global factors will put their mark on the perceptual organization of these displays. The gist of this idea will be made more clear by an analysis of the patterns of Figure 7 .

Figures 7B and 7C demonstrate completion interpretations of Figure 7A. Interpretation 7B exploits the advantage of good continuation: the sides of the partly occluded surface, $S$, continue perceptually without any information behind the occluding surface. In interpretation $7 \mathrm{C}$, the local advantage that a completion interpretation may have is not exploited. Therefore, according to the hypothesis formulated above, interpretation $7 \mathrm{C}$ will not be perceptually preferred. Figures $7 \mathrm{D}$ and $7 \mathrm{E}$ each illustrate a mosaic interpretation of Figure $7 \mathrm{~A}-\mathrm{a}$ cross next to a square. Interpretation 7D exploits the advantage of good continuation in describing the common contour: the sides of the concave angle, $a$, are perceived as continuing inward. By doing so, the concave angle is removed from the description and the cross is perceptually divided into parts at concave angles. There are good reasons to believe that people do in fact organize shapes perceptually by decomposing them into parts at concave cusps (Hoffman \& Richards, 1984; Koenderink \& van Doorn, 1982). Although Buffart et al. (1981) did not present interpretations in which figures were represented by a number of smaller figures, there is nothing in coding theory itself that runs counter to it (Boselie \& Leeuwenberg, 1985). In interpretation $7 \mathrm{E}$, the sides of the cross that give shape to the common border are not continued perceptually. Therefore, according to the hypothesis, this interpretation will not be perceptually salient. In sum, interpretations 7C and 7E will not be perceptually attractive.

With respect to interpretations 7B and 7D, which both exploit good continuation, preference will be stronger for the interpretation that provides the globally simplest shape. In other words, preference will be strongest for the interpretation in which good continuation results in the simplest shape of the figure accompanying the square. The information of the partly occluded shape of Figure 7B amounts to $I=6$. The information of the nonoccluded cross, partitioned as illustrated in Figure 7D, amounts to $I=5$. According to the hypothesis, Figure 7A thus will be preferentially interpreted as a mosaic and not as an instance of occlusion. To summarize, I conjecture that with this kind of display, a global minimum principle operates only within the constraints of locally minimal descriptions.

The hypothesis formulated above states that in completion interpretations, as well as in mosaic interpretations based on partitioning, shapes are "invented" that show good continuation either at points of intersection or at the common contour. The hypothesis, however, does not specify how the contour of such invented shapes is closed beyond the points of intersection or the common contour. With respect to completion interpretations, will completion beyond the points of intersection result in a figure that is globally the simplest one, which may require the introduction of new angles somewhere along the invented contours? Or will things be kept locally as simple as possible; that is, will the sides behind the occluding figure be continued until they butt against each other, without introducing new angles somewhere on the way? See Figure 8 for an example of this kind of situation.

My guess is that if an interpretation combines a high degree of global simplicity with the local advantage of good continuation, this combination will be preferred perceptually over other interpretations that are globally even simpler but lack good continuation. However, if a high degree of global simplicity is incompatible with the local advantage of good continuation, perceptual ambiguity will result. These conjectures are specified more fully in the Predictions section.
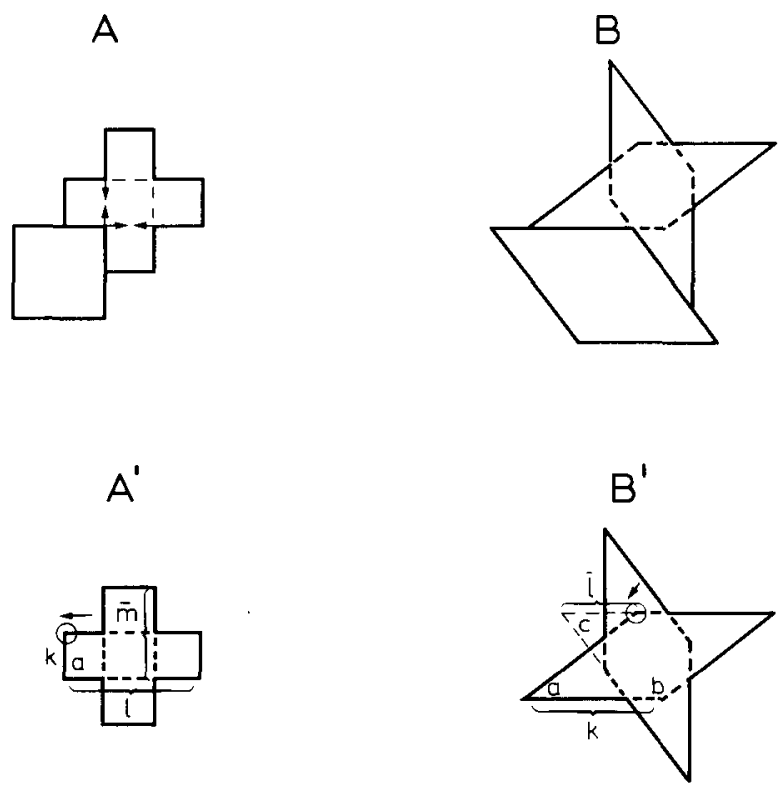

$$
\begin{gathered}
e^{*}\left(\langle a \rangle \left\langlee^{*}(k \quad l)\right.\right. \\
\left.\left.e^{*}(\&) 2^{*}(\bar{m})\right\rangle\right) \\
I=5
\end{gathered}
$$

$$
e^{*}\left(\left\langle e^{*}(\langle k\rangle\langle a \quad b\rangle) c\right\rangle\langle\bar{l}\rangle\right)
$$$$
I=5
$$
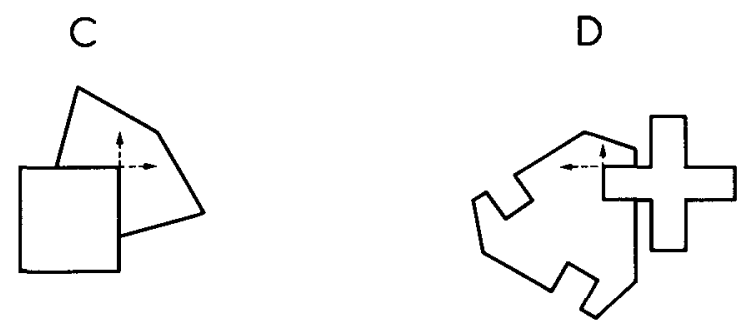

Figure 9. A and B: Contours that are continued inward butt against other lines, starting from other concave angles. $A^{\prime}$ and $B^{\prime}$ : Codes of parts of the Patterns $A$ and $B$, made in accordance with the hypotheses tested here. $C$ and $D$ : Examples of patterns in which the continued contours of concave angles do not meet other virtual lines emanating from concave angles, but do split up sides of the figure in question. According to the hypothesis tested here, Patterns $C$ and $D$ will not be interpreted as mosaics. 


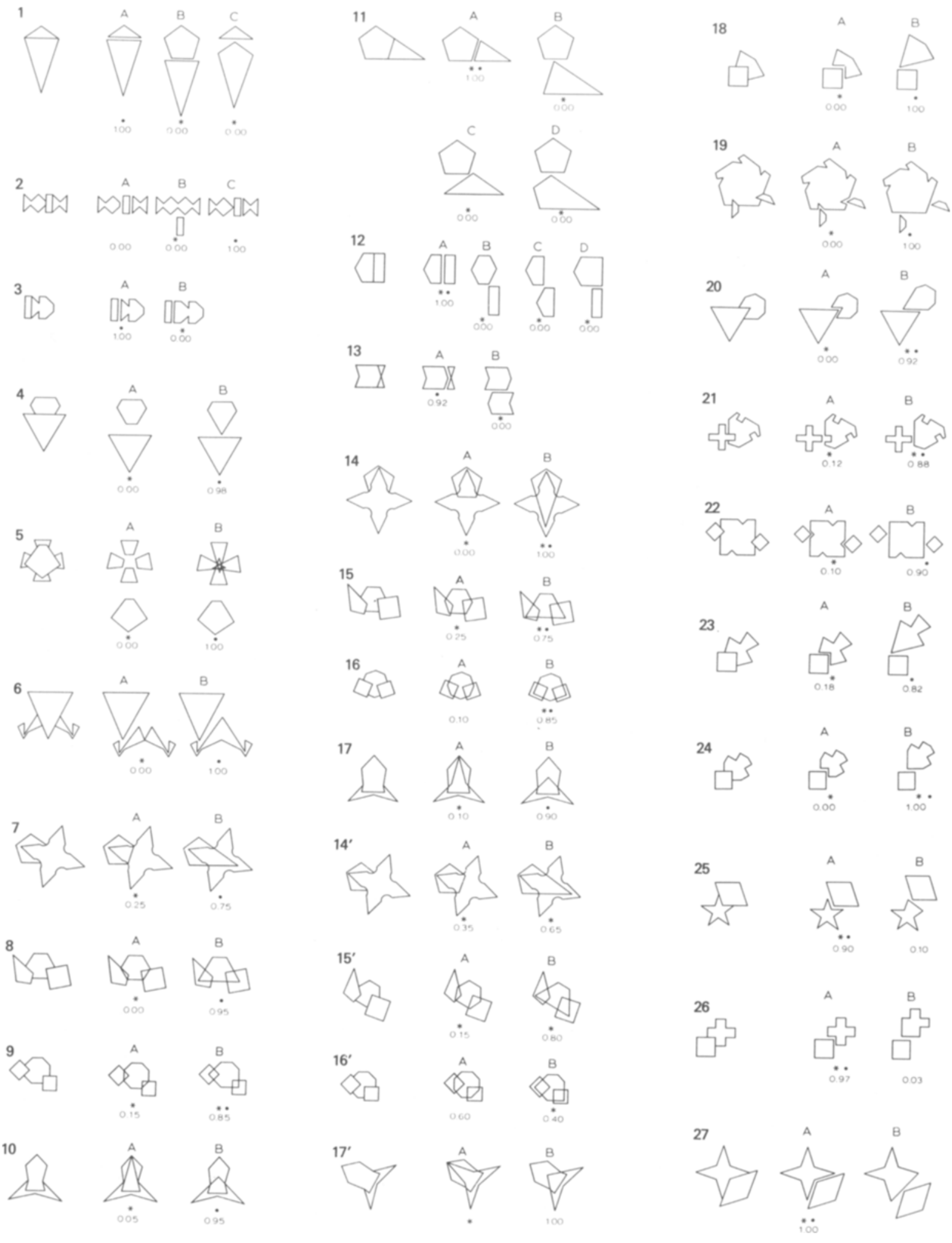

Figure 10. The 27 patterns used in the experiment reported here (left column), together with an illustration of the perceptually preferred interpretations $(A, B, C, \ldots$. Interpretations predicted by Buffart et al. (1981) are indicated by an asterisk(*); those predicted by the hypotheses tested here are indicated by a black dot $(\bullet)$. The codes of the different interpretations, made according to the coding procedure as used by Buffart et al., are available from the author on request. Figures 5,8 , and 9 present examples of coding analyses. Below each interpretation is given the proportion of subjects who actually preferred the interpretation. If the proportions do not add up to 1.0 , it is because of the occurrence of other, idiosyncratic responses, which are not shown here. For all patterns, the number of subjects is 60, except for patterns $7-10,14-17$, and $14^{\prime}-17^{\prime}$, which were presented to 20 subjects. 
As regards mosaic interpretations based on partitioning, sides that continue inward must end before they split up another side or an angle of a pattern because, in that case, information would increase locally. Of course, this would not be attractive. Therefore, there will be a preference to close the contour of an invented surface by continuing the sides of a concave angle until they butt against other sides that are continued inward themselves. This line of thought implies that continuation of the sides of a concave angle of the central contour of meshing figures will be attractive only when these sides meet other sides which themselves result from the continuation of sides of another concave angle. Figure 9 gives some examples of partitioning. These considerations on the nature of preferred completion and mosaic interpretations can be specified into a number of predictions, which are presented in the next section.

\section{Predictions}

When a completion interpretation does not describe the sides of an occluded surface as continuing behind an occluding one, it will not be preferred perceptually. Note that this prediction does not imply, as the local-cue approach does, that a $\mathrm{T}$-intersection entails a completion interpretation. Three cases can be distinguished here.

1. When a pattern makes it impossible to continue any one side of a surface, $a$, behind another one, the perceptually preferred outcome will not be an occlusion of $a$. This prediction was tested with Patterns 1, 2, and 3 of the experimental set (see Figure 10). For all three patterns, Buffart et al.'s (1981) coding theory of completion predicts that completion interpretations will be preferred.

2. A completion interpretation can be represented by a code in which a side of an occluded surface that partly disappears behind an occluding figure is described by means of two lengths that end and start, respectively, at the point where the two surfaces meet in the drawing. This is exactly what Buffart et al. (1981) did when they made codes of completion interpretations. In some cases, the predictions based on these codes differ from those based on codes in which such a side is described as one continuing length. In those cases, Buffart et al.'s predictions, which were based on the interruption of sides at intersection points, will prove to have been wrong. In the present study, this prediction was tested with Patterns 4,5 , and 6 of the experimental set.

3. A completion interpretation that contains an angle at the point of intersection of the occluding and occluded surfaces will not be preferred perceptually. In the present study, this prediction was tested with Patterns 7, 8, 9, and 10. Buffart et al. (1981) predicted that, for these patterns, completion interpretations with angles at the points of intersection would be preferred, except for Pattern 9, for which they predicted perceptual ambiguity.

A special class of patterns is formed by drawings without $\mathrm{T}$-intersections. The sides of their figures are in a direct line with each other in both mosaic and completion interpretations. In these cases, the local advantage of continuity of direction can be obtained by a mosaic interpretation without the disadvantages of depth information and occluded contours, which are inherent to a completion interpretation.

4. I conjecture that in the cases of the patterns described above, a mosaic interpretation made up either of two surfaces or of one surface with a line in it will be preferred perceptually over a completion interpretation. In the present study, this prediction was tested with Patterns 11, 12, and 13. For Patterns 11 and 12, Buffart et al. (1981) predicted equal preferences for mosaic and completion interpretations; for Pattern 13, they predicted a preference for a completion interpretation.

The four predictions formulated above relate to the description of the contour of an occluded surface at points of intersection with an occluding surface. The next prediction relates to the way an occluded figure is completed after points of intersection have been crossed.

5 . With respect to the question of how the contour of an occluded surface is closed beyond the points of intersection, I have already made a distinction between two situations: When an interpretation combines a high degree of global simplicity with the local advantage of good continuation, it will be preferred over other interpretations that are globally even simpler but lack good continuation. However, when a high degree of global simplicity is incompatible with the local advantage of good continuation, perceptual ambiguity will result. Of all economical ways to describe a pattern, mirror symmetry, especially along a vertical axis, produces a high degree of global simplicity and is by far the perceptually most salient one (Barlow \& Reeves, 1979; Perkins, 1976). There will always be a strong tendency toward a completion that preserves the symmetry along the vertical axis, if already present, or that closes the partly occluded object in a symmetrical way. My guess, therefore, is that a completion interpretation that combines vertical symmetry with good continuation will be perceptually most salient. This prediction was tested by Patterns 4, 5, 6, 14, 15, 16, and 17. In each of these patterns, the occluded surface can be completed in at least two ways, both of which contain the same axis of symmetry. In one of the completions, the sides of the partly occluded object that disappear behind the occluding surface are continued until they meet each other. This solution is locally simple. In the other occlusion interpretation, the occluded object is completed by the introduction of new angles and sides that deviate from the directions of the sides that are actually drawn. Thus, regular surfaces are formed in Patterns 14-17: a pentagon (Pattern 14), a hexagon (Pattern 15), an octagon (Pattern 16), and a star (Pattern 17). All these surfaces have several axes of symmetry, in contrast to the shapes formed by the continuation of sides, which have one axis of symmetry only. The regular polygons lack the local simplic- 
ity of good continuation of sides. When the patterns are oriented in such a way that the axis of symmetry common to both interpretations is vertical (Patterns 14-17), both interpretations display the global simplicity of vertical symmetry, but only the first has the additional advantage of being locally simple as well. These interpretations will be the perceptually preferred ones.

When the orientation of the patterns is changed in such a way that the regular polygons do have a vertical axis of symmetry and the less regular shapes of the locally simple interpretations do not (the ' version), there will be a rivalry between the global simplicity of the regular solutions and the local attractivity of the less regular ones. In these cases, either interpretation could be preferred. The predictions made by Buffart et al. (1981), however, are independent of the orientation of patterns. According to the coding theory of visual completion, both types of completion interpretations are equally attractive in the case of Patterns 14 and 15; for Pattern 16, the prediction is that the completion with local simplicity will be preferred; for Pattern 17, the completion that lacks local simplicity will be the perceptually preferred one.

6. If there is an angle somewhere along a common contour, and if the sides of the concave angle, when continued inward, do not meet a side emanating from another concave angle, then a mosaic interpretation will not be preferred over a completion interpretation, although globally it would be more simple than a completion. This prediction was tested with Patterns 18, 19, 20, and 21 . Coding theory predicts here that subjects will prefer a mosaic interpretation for Patterns 18 and 19, whereas Patterns 20 and 21 will be perceptually ambiguous.

7. If a mosaic interpretation can be made that is locally simple-that is, when the sides of the common contour are continued inward and meet other sides which themselves result from the continuation of sides of other concave angles-this interpretation, nevertheless, will not be preferred over a completion interpretation when it lacks global simplicity. This prediction was tested with Patterns 22,23 , and 24 . Coding theory predicts that subjects will prefer a mosaic interpretation for Patterns 22 and 23, whereas Pattern 24 will be perceptually ambiguous.

8. If a mosaic interpretation can be made that is both globally and locally simple, then it will be preferred over completion interpretations. This prediction was tested with Patterns 25, 26, and 27. Coding theory also predicts mosaic interpretations for these patterns.

These predictions were tested in the experiment reported below.

\section{METHOD}

\section{Subjects}

Sixty students of educational psychology participated in the experiment.

\section{Materials}

The 31 patterns shown in Figure 10 were used in the experiment. Of the 31 patterns, three subsets (Patterns 7-10, 14-17, and 14'-17') are very similar to each other. To prevent the choice of an interpretation for a pattern from being affected by the way another very similar pattern had already been interpreted, each subject was presented with only one of these subsets. Each subject thus saw 23 patterns. The three subsets mentioned above were presented to three different groups of 20 subjects; the other 19 patterns were presented to all subjects $(N=60)$.

\section{Procedure}

Essentially, the same procedure as that used by Buffart et al. (1981) was followed. The subjects were run individually. In the instructions, three of the patterns of the set used by Buffart et al. (their Displays 1, 5, and 15) were shown. The subjects were told that every drawing represented two or more surfaces. They were asked to look carefully at each pattern and to tell the experimenter what they saw: a pattern made up of figures, with one figure partly occluding another, or a pattern made up of figures all in the same plane. When an occlusion interpretation was reported, the subjects were asked to indicate the exact form of the figure behind the occluding one. Next, they were shown how their interpretation of a pattern could be indicated on the drawing by tracing the contours of the figures they saw.

To produce a different random order for each subject, the 23 patterns, each on a separate sheet of paper, were shuffled before being presented. The subject looked at the drawing that lay before $\mathrm{him} /$ her on a table at a normal viewing distance. Each pattern was presented for $5 \mathrm{sec}$. If by that time the subject had not yet started to trace the contours of the figures that, in his or her perception, made up the pattern, he or she was asked to come to a decision. This time limit was introduced because our interest was in perception as a rapid, effortless process, in contrast to the process of scrutinizing a stimulus. As Julesz (1971) noted, any kind of regularity that is presented in a pattern can be detected after the expenditure of sufficient effort and time. But as our interest was in the result of the process of spontaneous perceptual organization, it was deemed advisable to limit the inspection time.

\section{RESULTS}

The subjects nearly always drew their interpretations within a few seconds after the presentation of a pattern. The interpretations, together with the proportion of subjects who drew them, are given in Figure 10.

\section{Testing the Predictions}

Predictions 1, 2, and 3 all forbid completion interpretations that do not continue sides of occluded figures without interruption behind occluding ones. The experimental results strongly support this rule: of the 440 interpretations given on Patterns 1-10, only $9(2 \%)$ broke this rule. The $2 \%$ of the responses are as predicted by Buffart et al.'s (1981) theory of visual completion. The remaining $98 \%$ are incompatible with their theory.

Prediction 4 pertains to a special case, in which a completion interpretation is less attractive because the advantage of continuity of direction can be obtained by a mosaic interpretation. The prediction was borne out by the experimental results: not even one completion interpretation was given as a response to Patterns 11-13. Since coding theory predicts equal preferences for a mosaic interpretation and three different completion interpretations (Patterns 11 and 12) or a preference for a comple- 
tion interpretation (Pattern 13), these results present strong evidence against Buffart et al.'s (1981) theory.

Prediction 5 was tested by the responses on seven patterns (Patterns 4, 5, 6, 14, 15, 16, and 17). Of the 260 interpretations given on these patterns, $250(96 \%)$ were as predicted here, whereas only $59(23 \%)$ were as predicted by Buffart et al.'s theory.

The results changed when the orientation of Patterns 14-17 was changed to favor a rivalry between interpretations that were either globally or locally simple. Although the predictions made by Buffart et al. (1981) were independent of the orientation of a pattern, $60 \%$ of the responses given on Patterns $14^{\prime}-17^{\prime}$ were as predicted by their theory, in contrast to $23 \%$ of the responses given on Patterns 14-17.

Prediction 6 states that when the disadvantage of an angle on a common contour cannot be avoided by the partitioning of the concave angle, a mosaic interpretation will not be preferred. This prediction was supported by $97 \%$ of the responses on the four patterns by which it was tested (Patterns 18-21). These results are incompatible with Buffart et al.'s (1981) predictions for these patterns.

Prediction 7 was tested by the responses on Patterns 22-24. Ninety percent of the interpretations given by the subjects confirmed the claim that a locally simple mosaic interpretation would not be preferred over a completion interpretation when it lacked global simplicity. Only $43 \%$ of the responses on these patterns were as predicted by coding theory.

Prediction 8, which states that mosaic interpretations that are both locally and globally simple will be preferred over completion interpretations, was supported by $96 \%$ of the responses given on Patterns 25-27. These results also fit the predictions based on Buffart et al.'s (1981) theory of completion.

To conclude, I will sum up how well the predictions of Buffart et al.'s (1981) theory are supported by the results. Of the 27 different patterns presented in the experiment (Patterns 14'-17' differed from Patterns 14-17 only in orientation), 18 patterns, according to coding theory, were theoretically unambiguous and 9 were theoretically ambiguous. For 14 of the 18 unambiguous patterns, the preferred interpretation as predicted by coding theory differed from the one predicted by this study. In none of these cases did the results support coding theory. Of the 720 responses given to these 14 patterns, only $25(4 \%)$ were as predicted by Buffart et al.'s theory. For the 9 patterns that were theoretically ambiguous, coding theory predicted that responses would be distributed over the equivalent interpretations. However, for 5 of these patterns, the responses were not distributed over theoretically equivalent interpretations at all: all subjects preferred the same interpretation! The distribution of the responses on the 4 remaining patterns was always very skewed, with Pattern 15 being an exception.

\section{Tests of Other Approaches}

Familiarity. From the point of view of familiarity, it might be predicted that subjects would prefer completions that formed familiar figures. Though no measure of familiarity is available, it seems not very plausible to claim that the preferred interpretations of Patterns 9, 21, or 24, to mention just a few patterns, contain figures that are more familiar to the average subject than the perceptually nonpreferred ones. On the contrary, the figures of the nonpreferred interpretations of these patterns look more familiar, at least in the sense that they are more simple and regular, than the ones that are actually preferred perceptually.

Aside from this, it so happens that two interpretations of a pattern are alike in that the figures they describe have the same shape. The only difference is one of area (see Pattern 11). Although these interpretations thus do not differ in terms of the familiarity of the figures they describe, one of them is nonetheless preferred perceptually.

Local configurations. According to a local-cue approach, a local cue, such as a T-intersection, favors a completion interpretation. If one contour continues through an intersection and another contour is cut off, an interpretation in which the continuous contour belongs to a figure in front will be preferred. This hypothesis, however, cannot explain the results for Patterns 25-27, nor does it make clear why Patterns 4-10 were preferentially completed in a particular way.

Good continuation. The Gestalt principle of good continuation claims that the perceptual system prefers to continue directions. Although this hypothesis does a good job in predicting the particular completions made on many patterns, it is disproved as a general rule of perceptual organization by the results of Patterns $14^{\prime}$ and $16^{\prime}$. Additionally, this principle cannot explain the results for the type of pattern represented by Patterns 1-3 and 25-27.

Good Gestalt. This approach emphasizes that perceptual organization will result in "good" figures, figures that embody such properties as regularity, symmetry, simplicity, unity, and homogeneity. This hypothesis is contradicted by the results of many patterns of the experimental set. The figures that formed part of the preferred interpretations were very often less regular than the figures of less preferred interpretations (see Patterns 1-4, 7-10, and 21-24).

Rock's coincidence principle. Because Rock (1983) did not formulate a metric of the "coincidenticity" of an interpretation, interpretations cannot be ordered in an unambiguous way according to their power to explain seeming coincidences. Take Patterns 1 and 11 . All interpretations of these patterns contain surfaces with coinciding contours. So, on the face of it, none of them will be expecially attractive. Nonetheless, with both patterns, there is a strong preference for one interpretation over all others. Or consider the two interpretations of Pattern 5: neither interpretation describes a seeming coincidence, and yet one of them is strongly preferred over the other. Aside from the problem of a metric of coincidenticity, the coincidence principle, as it was applied by Rock in the examples he produced to support it, is disproved by the results of Patterns 25-27. 


\section{DISCUSSION}

Many students of perception (Arnheim, 1954; Kopfermann, 1930; Rock, 1983) hold the view that there is a strong tendency to avoid interpretations in which two regions are shaped by a double-sided contour. In their opinion, all mosaic interpretations are perceptually unattractive and probably even impossible to attain (see Kennedy, 1974, for a lengthy discussion). However, according to Buffart et al. (1981), mosaic and completion interpretations are equally attractive in principle. The only thing that matters is the information load of a code, irrespective of the kind of interpretation it represents.

In this study, a distinction was drawn between two kinds of patterns: patterns that had only straight lines as common contours versus patterns with an angle as part of a central contour. Straight lines as common contours were not detrimental to a mosaic interpretation (Patterns 1-3 and 11-13), but angles on a common contour were, unless they could be avoided by means of a globally simple partitioning code that exploited the local advantage of continuity of sides (Patterns 25-27). So, in my opinion, the view that mosaic interpretations per se are unattractive, as well as Buffart et al.'s claim that the information load of codes is the only decisive factor in perceptual preference, is wrong. In addition to the experimental evidence produced in this study, analyses of other experiments lend support to this point of view. Below are two examples.

Of the 25 patterns used by Buffart et al. (1981), 11 patterns had only straight lines as common contours, whereas 11 other patterns had an angle on the central contour. In just one case (Pattern 13 of Buffart et al.'s set) could the concave angle of the common contour be avoided by a globally simple partitioning code. For the 10 other patterns with a central angle, a mosaic interpretation is unattractive according to the rules proposed by this study. Of the responses given by the subjects in Buffart et al.'s experiments on this last subset of patterns, only $7 \%$ were mosaic interpretations, in contrast to $37 \%$ mosaic interpretations on the former subset. Eighteen of the patterns used by Chapanis and McCleary (1953) were of the same type as those used by Buffart et al. Of the 18 patterns, 6 had straight lines as common contours and 12 had angles as part of the central contour. For 11 of the 12 patterns with a central angle, a mosaic interpretation is unattractive according to the rules proposed by this study. Of the responses given by Chapanis and McCleary's subjects on this last subset, only $17 \%$ were mosaic interpretations, whereas the former subset elicited $60 \%$ mosaic interpretations. These data once more support the view that observers preferentially avoid mosaic interpretations that do not exploit the local advantage of continuity of sides and that thereby describe angles on the common contour twice.

A striking difference between coding theory and the approach advocated here is that coding theory marks a substantial number of the patterns presented in the experi- ment $(N=9)$ as being theoretically ambiguous, whereas the present approach does not $(N=1)$. This applies with even greater force to the set of patterns Buffart et al. (1981) used to test their theory. Of the 25 patterns they presented, as many as 15 were theoretically ambiguous as defined by their coding procedure. That is, of the several different ways to interpret these patterns, at least some interpretations had the same minimal information load. This should have resulted in a distribution of responses over the equivalent interpretations. According to Buffart et al., such a prediction of perceptual ambiguity would provide a strong test of coding theory (1981, p. 266). With many theoretically ambiguous patterns, however, the response frequencies produced by the subjects were very skewed. For 10 of the 15 patterns in question, the response frequencies found differed in a significant way (binomial test) from the ones expected if these patterns were really perceptually ambiguous as defined by coding theory. In their 1983 article, Buffart et al. presented two models to describe the response frequencies of the 15 theoretically ambiguous patterns. These models started from the assumption that these patterns were perceptually ambiguous and that the departures from the theoretically expected frequencies were due to the subject's strategy of escaping ambiguity by making a number of supplementary interpretations. However, when Buffart et al.'s set of patterns is described according to the approach advocated in this study, their complicated analysis of ambiguity becomes completely superfluous: now none of their patterns turn out to be theoretically ambiguous. The question then arises: How well does the present approach succeed in describing their experimental results?

Starting from the considerations on the nature of mosaic and completion interpretations given above, Buffart

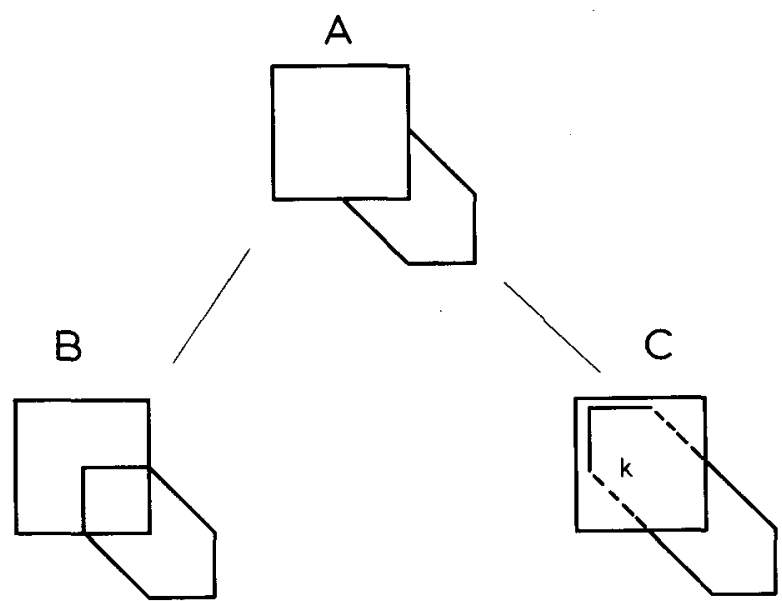

Figure 11. A: Pattern 7 of Buffart et al.'s (1981) set (see Figure 3). B: The preferred interpretation of Pattern A, as predicted by Buffart et al. C: The set of preferred interpretations, as predicted by the hypotheses tested here. The length $k$ is variable. $B$ and $C$ differ only in the elongation of the predicted figures. 
et al.'s (1981) set of patterns falls apart into two groups. First of all, they presented a number of patterns in which part of the contour of the square and of the figures accompanying it were in a direct line, thus forming two continuing lines that intersected at a corner of the square (Patterns $4,6,14,15,16,19,20$, and 24). From the point of view of the present approach, these patterns form a special case: (1) In the completion interpretations, the corners of the occluding figure (the square) and the occluded figure coincide, and (2) in the mosaic interpretations, the locally very simple crosslike configuration does favor the interpretation of the lines as wires, and not as contours of surfaces. Another special set of patterns is formed by displays in which the sides of the occluded figure that disappear behind the occluding one are parallel to each other (Patterns 7, 12, 16, and 22). In those cases, the drawing-in response may not have been suitable for the task of describing the way in which the occluded figure was initially completed perceptually. I will come back to this point later.

In sum, the present approach suggests that two groups of patterns should be kept apart in the analysis of Buffart et al.'s (1981) results: the 11 patterns that form special cases (Patterns 4, 6, 7, 12, 14, 15, 16, 19, 20, 22, and 24 ) and the 14 remaining patterns. Of the responses given on this last group, $83 \%$ were as predicted by the present approach. Of the responses given on the 11 special cases, $44 \%$ were as predicted by this study. The result of this last group of patterns was especially negatively affected by Patterns 7,22 , and 24 . Whereas the preference for the completion interpretation of Pattern 24 reported by Buffart et al. cannot be understood from the analysis given here, the negative results on Patterns 7 and 22 were probably due to the difference between perceptually completing an occluded figure and representing the completion in question by means of a drawing. This seems a plausible explanation, because the only difference between the predicted and the actually drawn figure is an elongation of the figure (see Figure 11). When illustrating their completion of these patterns, the subjects very often started the closure of the occluded figure by making an angle at the point where the occluding and occluded figure met. In my opinion, however, it is questionable whether the subjects really wanted to indicate thereby that they had seen the contour of the occluded figure change direction at exactly that point. Because Buffart et al. did not stress this point in their instructions to the subject, this question remains open.

These analyses of Buffart et al.'s (1981) data, along with the results of the experiment reported here, strongly suggest that, contrary to Buffart et al.'s claim, a global minimum principle in perception operates only within the constraints set by several local minima. The relevance of the local minima that have been dealt with here has to be corroborated by further research.

A final remark is in order about the application of Leeuwenberg's $(1969,1971)$ coding system here. The aim of the present reseach was to refute Buffart et al.'s (1981) claim that a global minimum, as specified by Leeuwenberg's coding system, answers the questions raised by the phenomenon of visual completion. In order to oppose their view, I had to apply their own set of coding rules. Obviously, this does not mean that, in my opinion, the coding system itself is above criticism.

\section{REFERENCES}

ARnheIm, R. (1954). Art and visual perception. Berkeley: University of California Press.

Barlow, H., \& ReEves, B. (1979). The versatility and absolute efficiency of detecting mirror symmetry in random dot displays. Vision Research, 19, 783-793.

Boselie, F., \& LeEuWEnBerG, E. (1985). Birkhoff revisited: Beauty as a function of effect and means. American Journal of Psychology, 98, 1-39.

Boselie, F., \& LeEuwenberg, E. (1986). A test of the minimum principle requires a perceptual coding system. Perception, 15, 331-354.

Buffart, H., Leeuwenberg, E., \& Restle, F. (1981). Coding theory of visual pattern completion. Journal of Experimental Psychology: Human Perception \& Performance, 7, 241-274.

Buffart, H., LeEuWenberG, E., \& Restle, F. (1983). Analyses of ambiguity in visual pattern completion. Journal of Experimental Psychology: Human Perception \& Performance, 9, 980-1000.

Chapanis, A., \& MCCleary, R. (1953). Interposition as a cue for the perception of relative distance. Journal of General Psychology, 48, 113-132.

Collard, R., \& Leeuwenberg, E. (1981). Judged temporal order and spatial context. Canadian Journal of Psychology, 35, 323-329.

DinNERSTEIN, D., \& WERTHEIMER, M. (1957). Some determinants of phenomenal overlapping. American Journal of Psychology, 70, 21-37.

GIBson, J. (1979). The ecological approach to visual perception. Boston: Houghton Mifflin.

Hatfield, G., \& Epstein, W. (1985). The status of the minimum principle in the theoretical analysis of visual perception. Psychological Bulletin, 97, 155-186.

HochberG, J., \& MCAlister, E. (1953). A quantitative approach to figural 'goodness.' Journal of Experimental Psychology, 46, 361-364.

Hoffman, D., \& Richards, W. (1984). Parts of recognition. Cognition, 18, 65-96.

Julesz, B. (1971). Foundations of cyclopean perception. Chicago: University of Chicago Press.

KANIZSA, G. (1975). The role of regularity in perceptual organization. In G. Flores d'Arcais (Ed.), Studies in perception (pp. 48-66). Florence: Martello.

Kanizsa, G. (1985). Seeing and thinking. Acta Psychologica, 59, 23-33.

KanizsA, G. (1986). Die Doppeldeutigkeiten der Prägnanz. Gestalt Theory, 8, 99-135.

KENNEDY, J. (1974). A psychology of picture perception. San Francisco: Jossey-Bass.

KoEnderink, J., \& VAN Doorn, A. (1982). The shape of smooth objects and the way contours end. Perception, 11, 129-137.

KoffKa, K. (1935). Principles of Gestalt psychology. New York: Harcourt, Brace.

KopfermanN, H. (1930). Psychologische Untersuchungen über die Wirking zweidimensionaler körperlicher Gebilde. Psychologische Forschung, 13, 292-364.

LEEUWENBERG, E. (1969). Quantitative specification of information in sequential patterns. Psychological Review, 76, 216-220.

LEEUWENBERG, E. (1971). A perceptual coding language for visual and auditory patterns. American Journal of Psychology, 83, 307-349.

LEEUWENBERG, E. (1982). The perception of assimilation and brightness contrast. Perception \& Psychophysics, 32, 345-352.

LEEUWENBERG, E., \& BufFART, H. (1984). The perception of foreground 
and background as derived from structural information theory. Acta Psychologica, 55, 249-272.

Perkins, D. N. (1976). How good a bet is good form? Perception, 5, 393-406.

RESTLE, F. (1979). Coding theory of the perception of motion configurations. Psychologial Review, 86, 1-24.

RESTLE, F. (1982). Coding theory as an integration of Gestalt psychology and information processing theory. In J. Beck (Ed.), Organization and representation in perception (pp. 31-56). Hillsdale, NJ: Erlbaum.

Rock, I. (1983). The logic of perception. Cambridge, MA: Bradford. VAN TuIJL, H. (1980). Perceptual interpretation of complex line patterns. Journal of Experimental Psychology: Human Perception \& Performance, 6, 197-221.
Van Tuill, H., \& Leeuwenberg, E. (1979). Neon color spreading and structural information measures. Perception \& Psychophysics, 25, 269-284.

Van Tuijl, H., \& Leeuwenberg, E. (1982). Peripheral and central determinants of subjective contour strength. In H. Geissler, P. Petzoldt, H. Buffart, \& Y. Zabrodin (Eds.), Psychophysical judgment and the process of perception (pp. 150-163). Amsterdam: NorthHolland.

(Manuscript received December 4, 1986; revision accepted for publication October 16, 1987.) 